\title{
A practical and sensitive method of quantitating lymphangiogenesis in vivo
}

\author{
Mousumi Majumder ${ }^{1}$, Xiping Xin ${ }^{1}$ and Peeyush K Lala ${ }^{1,2,3}$
}

To address the inadequacy of current assays, we developed a directed in vivo lymphangiogenesis assay (DIVLA) by modifying an established directed in vivo angiogenesis assay. Silicon tubes (angioreactors) were implanted in the dorsal flanks of nude mice. Tubes contained either growth factor-reduced basement membrane extract (BME)-alone (negative control) or BME-containing vascular endothelial growth factor (VEGF)-D (positive control for lymphangiogenesis) or FGF2/VEGF-A (positive control for angiogenesis) or a high VEGF-D-expressing breast cancer cell line MDA-MD-468LN (468-LN), or VEGF-D-silenced 468LN. Lymphangiogenesis was detected superficially with Evans Blue dye tracing and measured in the cellular contents of angioreactors by multiple approaches: lymphatic vessel endothelial hyaluronan receptor-1 (Lyve1) protein (immunofluorescence) and mRNA (qPCR) expression and a visual scoring of lymphatic vs blood capillaries with dual Lyve1 (or PROX-11 or Podoplanin)/Cd31 immunostaining in cryosections. Lymphangiogenesis was absent with BME, high with VEGF-D or VEGF-D-producing 468LN cells and low with VEGF-D-silenced 468LN. Angiogenesis was absent with BME, high with FGF-2/VEGF-A, moderate with 468LN or VEGF-D and low with VEGF-D-silenced 468LN. The method was reproduced in a syngeneic murine $\mathrm{C} 3 \mathrm{~L} 5$ tumor model in $\mathrm{C} 3 \mathrm{H} / \mathrm{HeJ}$ mice with dual Lyve1/Cd31 immunostaining. Thus, DIVLA presents a practical and sensitive assay of lymphangiogenesis, validated with multiple approaches and markers. It is highly suited to identifying pro- and anti-lymphangiogenic agents, as well as shared or distinct mechanisms regulating lymphangiogenesis vs angiogenesis, and is widely applicable to research in vascular/tumor biology.

Laboratory Investigation (2013) 93, 779-791; doi:10.1038/labinvest.2013.72; published online 27 May 2013

KEYWORDS: angioreactor; breast cancer; DIVLA; lymphangiogenesis; lymphatic metastasis

The study of lymphangiogenesis is an emerging field of research for defining the fundamental mechanisms of lymphatic vessel growth and function under physiological and pathological conditions. ${ }^{1,2}$ This knowledge serves as the key to therapeutic applications for both blocking and promoting lymphangiogenesis. For example, an association between intra- or peritumoral lymphangiogenesis with lymphatic metastasis in many cancers ${ }^{3-5}$ suggests that newly formed lymphatics serve as conduits for lymphatic invasion and spread of cancer cells to lymph nodes. This is often the first route of spread in many cancers that can subsequently metastasize from the lymph nodes to other organs via the blood stream. Thus, inhibition of lymphangiogenesis might offer a new opportunity for intervention of metastasis. Conversely, dysfunction of lymphatic vessels often leads to primary and secondary lymphedema, which markedly impairs the function of affected tissues or organs. Thus, delivery of lymphangiogenic factors to these affected tissues or organs might restore their functions by establishing functional lymphatic networks. ${ }^{6}$ Research on lymphangiogenesis was long limited by the lack of lymphatic-specific markers and suitable experimental models. Anatomical identification of the lymphatic system in small animals, generation of transgenic mouse models and recent in vitro models of culturing lymphatic endothelial cells (LECs) or lymphatic vessel explants have all paved the way for a new era in this research. ${ }^{7}$

Numerous in vitro systems have been developed to study lymphangiogenesis utilizing LECs from various sources: ${ }^{7}$ (a) lymphatic-rich lesions induced by injecting Freund's adjuvant, (b) explants of thoracic duct fragments, (c) LEC differentiation induced in embryoid bodies and (d) immune-purified primary

\footnotetext{
${ }^{1}$ Department of Anatomy and Cell Biology, Schulich School of Medicine and Dentistry, University of Western Ontario, London, Ontario, Canada; ${ }^{2}$ Department of Oncology, Schulich School of Medicine and Dentistry, University of Western Ontario, London, Ontario, Canada and ${ }^{3}$ Child Health Research Institute, Schulich School of Medicine and Dentistry, University of Western Ontario, London, Ontario, Canada

Correspondence: Dr PK Lala, MD, PhD, Department of Anatomy and Cell biology, Schulich School of Medicine and Dentistry, University of Western Ontario, London, Ontario N6A5C1, Canada.E-mail. pklala@uwo.ca 
or immortalized human dermal LEC. LECs, when placed on collagen gel or matrigel, align themselves to form tube-like structures under appropriate stimuli. ${ }^{8}$ However, most of these systems suffer from limitations including the short life or limited number of primary cells, or possible non-physiological nature of immortalized cells. In a more physiological, organotypic model of thoracic duct rings explanted in collagen gels allows lymphatic capillary-like sprouting under stimulated conditions, which can be quantified by computerassisted imaging. ${ }^{7}$ In our experience, this assay is time- and labor-intensive, requiring the death of many young mice of identical strain and sex for harvesting thoracic ducts, and low oxygen culture conditions that require standardization. None of these in vitro assays can adequately address the issues of realtime quantification of the growth of lymphatic vessels in vivo, with lumens and lymphatic stability, which are essential for the maintenance of lymphatic functions. For these reasons, a reliable, quick and powerful in vivo lymphangiogenesis assay is urgently needed.

Unlike blood vessels, lymphatics are not perfused with red blood cells and thus remain poorly visible in a given tissue. Their macroscopic detection is facilitated by the uptake of dyes such as Evans Blue or India ink from the extravascular space. ${ }^{9}$ At the microscopic level, immunohistochemical staining for specific markers such as lymphatic vessel endothelial hyaluronan receptor-1 (Lyve1), vascular endothelial growth factor receptor 3 (VEGFR3) or Podoplanin (Pdpn) or PROX-1 (Prox1) provides an opportunity for visualization of lymphatic microvessels. ${ }^{10-13}$ Examples of in vivo models manipulating lymphangiogenesis include (a) induction of tumor-associated lymphangiogenesis by VEGF-C or -D overexpression in tumor cells $^{8,14,15}$ or in transgenic mice, ${ }^{16,17}$ (b) lymphatic hyperplasia induced by intraperitoneal injection of incomplete Freund's adjuvant, ${ }^{18}$ (c) implantation of growth factor-containing pellets into corneal micropockets ${ }^{19,20}$ and (d) the use of avian chorioallantoic membrane (CAM) to stimulate lymphangiogenesis. ${ }^{21}$ Each assay has its own advantages and limitations depending on the objectives. However, some of them may include masking effects, for example, of native embryonic lymphangiogenesis in the CAM assay, or of inflammatory mediators produced by injection of incomplete Freund's adjuvant. Furthermore, many of these in vivo assays are time consuming and often qualitative, or at best, semiquantitative. To overcome these limitations, we have developed a quick, practical and quantitative in vivo assay for measuring lymphangiogenesis by implanting angioreactors in the mouse dorsal flanks. This method, an adaptation of the directed in vivo angiogenesis assay (DIVAA), ${ }^{22-24}$ is highly reproducible, providing an opportunity to quantify accurately lymphangiogenesis as early as 10 days. It is applicable to many objectives including quick preclinical screening of drugs in vivo. We name this method as 'directed in vivo lymphangiogenesis assay' (DIVLA). To illustrate its power, here we used a human breast cancer cell line MDA-MB468LN, expressing high levels of VEGF-D but little VEGF-C or
VEGF-A, and its VEGF-D-silenced derivative, to quantitate VEGF-D-dependent, tumor-associated lymphangiogenesis in nude mice. To reproduce DIVLA in immunocompetent mice, we used a syngeneic murine breast cancer cell line C3L5 that produced VEGF-C and -D in a cyclo-oxygenase (COX)-2dependent manner. ${ }^{25}$

\section{MATERIALS AND METHODS Human Cell lines}

Human breast cancer cell line MDA-MB-468LN (henceforth called $468 \mathrm{LN})$ is a lymphatic metastatic variant of the MDA-MB-468 line. ${ }^{26}$ Cells were used from the original stock shortly after their generation. We established stable VEGF-D knocked down 468LN cells using shRNA plasmids. ${ }^{8}$ Both 468LN- and VEGF-D-silenced 468LN cells (named $\Delta$ VEGF-D/468LNs) were grown as monolayers in $\alpha$-MEM supplemented with $10 \% \mathrm{FBS}, 50 \mathrm{U} / \mathrm{ml}$ penicillin and $50 \mu \mathrm{g} / \mathrm{ml}$ streptomycin (all materials from GIBCO/ Invitrogen, ON, Canada).

\section{Murine Cell Line}

C3L5 is a COX-2-expressing, PGE2-, VEGF-C- and VEGF-Dsecreting murine breast cancer cell line produced in our laboratory, the VEGF-C- and -D-secreting ability primarily attributed to COX-2 activity. ${ }^{25,27}$ Cells were maintained in high glucose DMEM (GIBCO, Grand Island, NY, USA) supplemented with $5 \%$ fetal bovine serum, $100 \mathrm{U} / \mathrm{ml}$ penicillin $\mathrm{G}$ and $100 \mu \mathrm{g} / \mathrm{ml}$ streptomycin in a humidified incubator with $5 \% \mathrm{CO}_{2}$ at $37^{\circ} \mathrm{C}$.

\section{Mice}

Four- to five-week-old athymic nude female mice (Hsd. Athymic Nude-Foxn $1^{n u} /$ Foxn $1^{+}$, Harlan, IN, USA) and C3H/ HeJ mice (obtained from the Jackson Laboratory) were allowed to acclimatize for 2 weeks, maintained, respectively, in barrier or traditional facilities, on standard mouse chow and tap water on a $12 \mathrm{~h}$ light/dark cycle and treated in accordance with the guidelines set by the Canadian Council on Animal Care.

\section{Directed In Vivo Lymphangiogenesis}

We significantly modified a directed in vivo angiogenesis assay $^{23}$ to measure lymphangiogenesis in addition to angiogenesis. Briefly, sterile angioreactors (Cat. No. 3450048-01; Trevigen, Gaithersburg, MD, USA) were prechilled at $4^{\circ} \mathrm{C}$ and filled with $20 \mu \mathrm{l}$ of either growth factor-reduced basement membrane extract (BME) (Cat. No. 3450-048-02; Trevigen) alone or $40 \times 10^{3} 468 \mathrm{LN}$ or $\Delta$ VEGF-D/468LN cells in $2 \mu \mathrm{l} \alpha$-MEM with $18 \mu \mathrm{l}$ BME, or $18 \mu \mathrm{l}$ BME with recombinant VEGF-D (20 ng in $2 \mu \mathrm{l})(622-\mathrm{VD}-005$; R\&D Systems, CA, USA) or FGF-2 (225 ng)/VEGF-A (75 ng) (Cat. No. 3450-048-B10; Trevigen), the latter two used as respective positive controls for lymphangiogenesis and angiogenesis. Angioreactors were incubated at $37^{\circ} \mathrm{C}$ for $1 \mathrm{~h}$ to allow BME gel formation, before subcutaneous implantation into the 
dorsal flank of 8-week-old female nude mice. Mice were anesthetized with isoflurane (Baxter, Mississauga, ON, Canada) and angioreactors (4 with identical conditions per mouse; 4-8 mice per condition; Figure 1) implanted in the dorsal flank. After the defined experimental period of 10 days, mice were anesthetized to expose the angioreactors. The skin surrounding the angioreactors was cut and opened with fine dissection scissors (Figure 1). Evans Blue was injected near the angioreactor to capture images of dye-stained lymphatics immediately and at short intervals up to $25 \mathrm{~min}$ with both a digital camera (Nikon, D-90) and a dissection microscope. Subsequently, mice were euthanized humanely to retrieve the angioreactors used for three different purposes (Figure 1). One of them was carefully removed without severing the ingrowing vessels and excising the rest of the tissues with fine scissors and flash-frozen immediately with dry ice for making cryosections. The other three were removed from the surrounding tissues by excising along the margins to sever any vessel that may be growing into them, and lifting them with dissection forceps. They were used to collect cellular contents and conduct lymphatic ingrowth assay and RNA extraction for real-time gene expression as detailed below.

\section{Evans Blue Dye Tracing}

Approximately $2 \mu \mathrm{l}$ of $4 \%$ Evans Blue dye (Sigma-Aldrich, San Francisco, CA, USA) diluted with PBS was injected subcutaneously in nude mice with a $30 \mathrm{G}$ needle at about $2 \mathrm{~cm}$ distance from the angioreactor implants, allowing a visual tracing of lymphatics growing into the angioreactors.

\section{Dorsal flank opened (Digital Camera)}

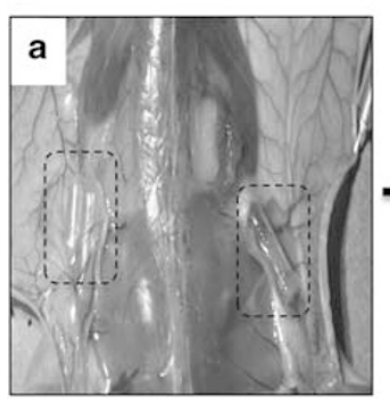

Evans Blue injected (Digital Camera)

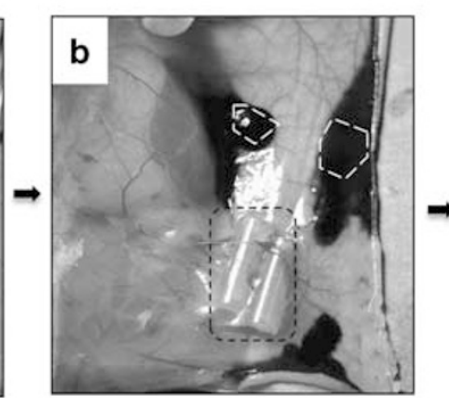

5 min post $\mathrm{EB}$ injection (Digital Camera)

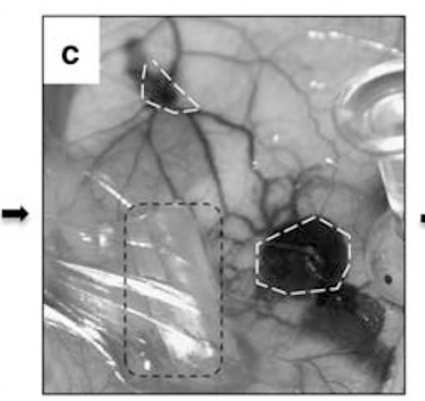

25 min post $\mathrm{EB}$ Injection (Dissection Microscope )

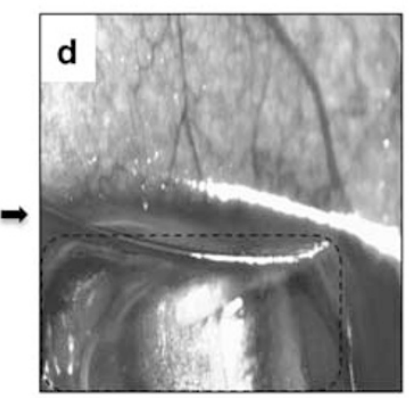

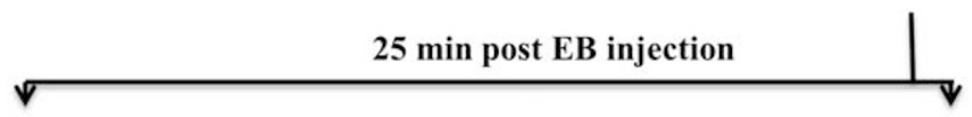

1 angioreactor collected with surrounding tissue without disturbing vascular ingrowth

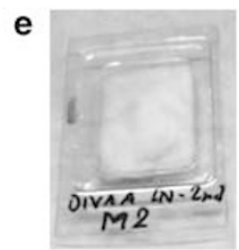

Flash frozen, cryosections were stained for Lyve1 (or Prox1 or Pdpn)/Cd31/DAPI

3 angioreactors removed from the surrounding tissues by excising along margins of tube

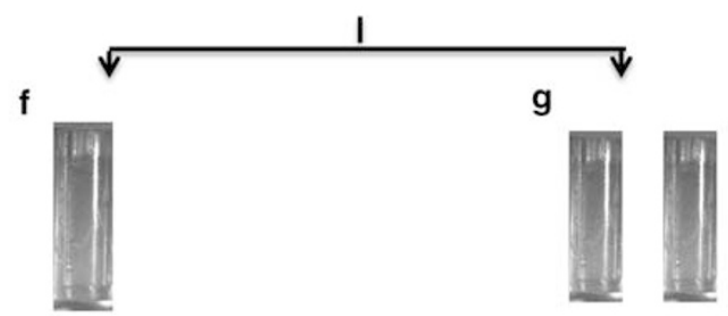

1 tube used for immunofluorescence assay for Lyve1
Cellular contents from 2 tubes pooled and RNA extracted to quantify Lyve1/Cd31/ $\beta$-actin mRNA

Figure 1 Workflow of directed in vivo lymphangiogenesis assay (DIVLA). Four angioreactors were implanted in one mouse. (a) After 10 days of implantation, mouse dorsal flank exposed to visualize angioreactors (black dotted line) and the surrounding vasculature. (b) Digital camera image (DCI) immediately following Evans Blue (EB) dye injection (white dotted line) at two to three points near the angioreactors. (c) DCl of EB drainage by lymphatics observed at 5 min intervals after injection; (d) image captured with dissection microscope at 5-min intervals up to 25 min after injection. (e) One angioreactor collected with intact surrounding tissue without disrupting the vascular ingrowth and embedded with OCT compound followed by flash freezing. The remaining three angioreactors were taken out to retrieve cellular contents. (f) Of the latter, one tube was used for immunofluorescence assay and (g) two were used for RNA extraction. DAPI, 4',6-diamidino-2-phenylindole. 
Images were captured at 5-25 min after injection under a dissection microscope (Nikon SMZ1500).

\section{Retrieval of Cellular Contents from Angioreactors}

Under stimulated conditions, lymphatics as well as blood vessels grew into the angioreactors, invading the BME. To quantitate lymphatic vessel ingrowth, angioreactors were removed from the surrounding tissues. The cellular contents were retrieved by cutting one end and squeezing the tube. The tubes were rinsed two times with CellSperse solution (Cat. No. 3450-048-05; Trivigen) to retrieve remaining tissue, which was incubated in $200 \mu \mathrm{l}$ of the solution for $1 \mathrm{~h}$ at $37^{\circ} \mathrm{C}$ to digest the BME. The incubation mix was then washed three times with wash buffer (Cat. No. 3450-048-03; Trivigen) and centrifuged ( 1500 r.p.m. for $5 \mathrm{~min}$ ) to get a BMEfree cell pellet. The pellet was resuspended in $500 \mu \mathrm{l} \alpha$-MEM supplemented with $10 \%$ FBS and incubated at $37^{\circ} \mathrm{C}$ for $1 \mathrm{~h}$ to allow cell surface recovery.

\section{Quantitation of Lymphatic Ingrowth}

Each cell pellet was treated with $400 \mu$ l DIVAA $1 \times$ Dilution Buffer (Cat. no. 3450-048-07; Trivigen) and rabbit antimouse Lyve1 antibody (Cat. No. 11-034; AngioBio, Del Mar, CA, USA; diluted 1:500) and incubated at $4{ }^{\circ} \mathrm{C}$ overnight. Then, cells were washed three times for 5 min with $1 \times$ TBST $(1 \times$ PBS $/ 0.3 \%$ Triton X-100) and treated with Alexa Fluor 594 (Invitrogen) anti-rabbit secondary antibody (1:5000 dilution) and incubated for another $1 \mathrm{~h}$. After three further washes, cells were resuspended in $200 \mu \mathrm{l}$ wash buffer and fluorescence was measured in a 96-well plate using a FLUOstar Omega (Bmg Labtech, Fisher Scientific, CA, USA) spectrofluorimeter (excitation $584 \mathrm{~nm}$, emission $620 \mathrm{~nm}$, gain $1500-3000)$. The mean fluorescence ( \pm s.e.) for 16 replicate assays was determined.

\section{Expression of Lymphatic and Vascular Endothelial Cell Marker mRNAs}

In our pilot study with four mice, we observed that the yield of mRNA extracted from cell pellets recovered from a single angioreactor was inadequate. Hence in our definitive experiments, we pooled the contents of two angioreactors from the same mouse (identical condition) (Figure 1). Total RNA was extracted from cell pellets using Qiagen RNeasy (Qiagen, Toronto, ON, Canada). Synthesis of cDNA and quantitative (q) RT-PCR was performed for murine Lyve1 (Mm00475056_m1), Cd31 (Mm01242584_m1) and $\beta$-actin (4352933E) genes with TaqMan Gene Expression Assays (Applied Biosystems).

\section{Direct Visualization and Measurements of Lymphangiogenesis and Angiogenesis}

The surgically excised tissue including the angioreactor was placed on a disposable vinyl specimen mold, which was then secured and flash frozen on dry ice with OCT compound (Tissue-Tek ${ }^{\star}$, Sakura Finetek USA, Torrance, CA, USA), and the samples were stored at $-20^{\circ} \mathrm{C}$ until further processing as follows. Angioreactors were cut longitudinally on a cryostat (Leica CM3050 S) into two halves to place the contents on specimen molds and covered with HistoGel (Thermo Fisher Scientific, Burlington, ON, Canada). Then, the molds were mounted with OCT and frozen again on dry ice. Subsequently, the molds were placed on a cryostat, sections cut at 5-6 $\mu \mathrm{m}$, and the serial sections picked up on a colorfrost plus slide (Fisher Scientific). Adjacent serial sections were separately stained with hematoxylin/eosin to identify cell morphology, and dual immunostained to visualize lymphatics (Lyve1 or Prox1 or Pdpn staining) and blood vessels (Cd31 staining). To evaluate possible macrophage staining with Lyve1 reported in some studies, ${ }^{28}$ sections were also doublestained for Lyve1 and F4/80. All the secondary antibodies and species-specific sera were from Life Technologies (Burlington, ON, Canada). Frozen sections were fixed in ice-cold acetone for $5 \mathrm{~min}$ at $4{ }^{\circ} \mathrm{C}$; all of the subsequent steps were performed at room temperature. Slides were rehydrated for $10 \mathrm{~min}$ in PBS, followed by incubation for $20 \mathrm{~min}$ at room temperature in blocking buffer (5\% normal goat serum in PBS with $0.3 \%$ Triton X-100) to block nonspecific antibody binding. Sections were then treated for $1 \mathrm{~h}$ with primary rat monoclonal Cd31 antibody, (MEC 13.3; Santa Cruz Biotechnology, Santa Cruz, CA, USA) 1:200, in dilution buffer PBST $(1 \times \mathrm{PBS} / 2 \% \quad \mathrm{BSA} / 0.3 \%$ Triton $\mathrm{X}-100)$. Following washing steps with blocking buffer, sections were treated with rabbit anti-mouse Lyve1 or Proxl (Acris Antibodies, San Diego, CA, USA), or Pdpn (Bioss, Wouburn, MA, USA) antibodies, 1:200, in dilution buffer in the dark for $2 \mathrm{~h}$ at room temperature. For macrophage staining, slides were incubated with Lyvel antibody, followed by rat anti-mouse F4/80 antibody (Abcam, Toronto, ON, Canada), 1:200, in the dark for $2 \mathrm{~h}$ at room temperature. Following three washes with $1 \times$ PBST, either Alexa Fluor 647 donkey anti-rabbit or goat anti-rabbit Alexa Fluor 594 secondary antibodies, 1:2000, were applied to detect Lyve1, Prox 1 and F4/80 in far red or red channel, respectively. On the other hand, for $\mathrm{Cd} 31$, sections were stained with either Alexa Fluor 594 Goat Anti-rat $\operatorname{IgG}(\mathrm{H}+\mathrm{L})$ to detect in red channel or with goat anti-rat Alexa Fluor 488 to detect in green channel. In the case of Pdpn, Alexa Fluor 594 donkey anti-rabbit and either Alexa Fluor 647 goat anti-rat or goat anti-rat Alexa Fluor 488 secondary antibodies were added in 1:2000 dilution to detect Pdpn in red and Cd31 in far red or green channel. Sections were incubated for $30 \mathrm{~min}$ at room temperature, washed with PBS, dried and mounted with Vectashield solution (Vector Laboratories, Burlington, ON, Canada). They were scanned at low magnification $(\times 250)$ under a BX51 microscope (Olympus) to identify the most vascular areas within the angioreactor. Exclusion of the primary antibody step was used as the negative control in each case to ascertain staining specificity. Representative images were taken with confocal microscope (Zeiss LSM510 META). Micro (blood) vessel density (MVD) and lymphatic 
vessel density (LVD) were assessed in dual-immunostained sections as reported. ${ }^{25,29}$ Three hotspots per section (areas of highest vascular density in the descending order, 24 per group) were identified and examined at $\times 400$ magnification. The incidence of marker-positive vascular-like structures (irrespective of complete or incomplete lumen, depending on the plane of section) per unit area was scored after setting the threshold for background, using the Image-J software (NIH, Bethesda, MD, USA). ${ }^{25}$ The mean values for MVD $v s$ LVD were computed as the staining indices for $\mathrm{Cd} 31$ (red) vs Lyve1 or Prox1 (far red, depicted as sky blue), or Cd31 (far red, depicted as sky blue) vs Pdpn (red). We had earlier shown that the 'hotspot' method provides results that are relatively very similar to those provided by the vascular density scores obtained by scanning the total area of the section. ${ }^{29}$

Following exactly the same procedure, $\mathrm{C} 3 \mathrm{H} / \mathrm{HeJ}$ mice received four angioreactors each containing BME and $40 \times 10^{3}$ C3L5 cells in shaven dorsal flank. Mice (two per treatment group) were treated for 9 days before being killed. Treatment regimens included Celecoxib $(125 \mathrm{mg} / \mathrm{kg}$ by oral gavage, two times daily), or $0.5 \%$ methylcellulose (Sigma), which served as the vehicle control. After 9 days, angioreactors retrieved from mice were subjected to dual immunofluorescent labeling for murine Lyve1 (green) and Cd31 (red) in frozen sections. The mean values for MVD and LVD were computed as the staining indices as described above.

\section{Statistics}

Data were analyzed using the Sigma Stat (version 3.5; Systat, San Jose, CA, USA). All parametric data were analyzed using a one-way ANOVA and a Tukey-Kramer comparisons post hoc test. A Student's t-test was used to compare two data sets. All statistical tests were two-sided, and data were considered statistically significant at $P<0.05$.

\section{RESULTS}

In this illustrative study, we used MDA-MD-468LN and $\triangle$ VEGF-D/468LN cells to examine the role of VEGF-D expression by tumor cells on tumor-induced lymphangiogenesis in vivo. VEGF-D secretion ( $24 \mathrm{~h}$, in serum-free medium) by these cells were, respectively, $229.50 \mathrm{pg} / \mathrm{ml}$ for $468 \mathrm{LN}$ and $48.33 \mathrm{pg} / \mathrm{ml}$ for $\Delta$ VEGF-D/468LN, measured by ELISA. ${ }^{8}$

In the illustrative study, tubes containing BME alone provided the negative control; those with recombinant

Time course post injection

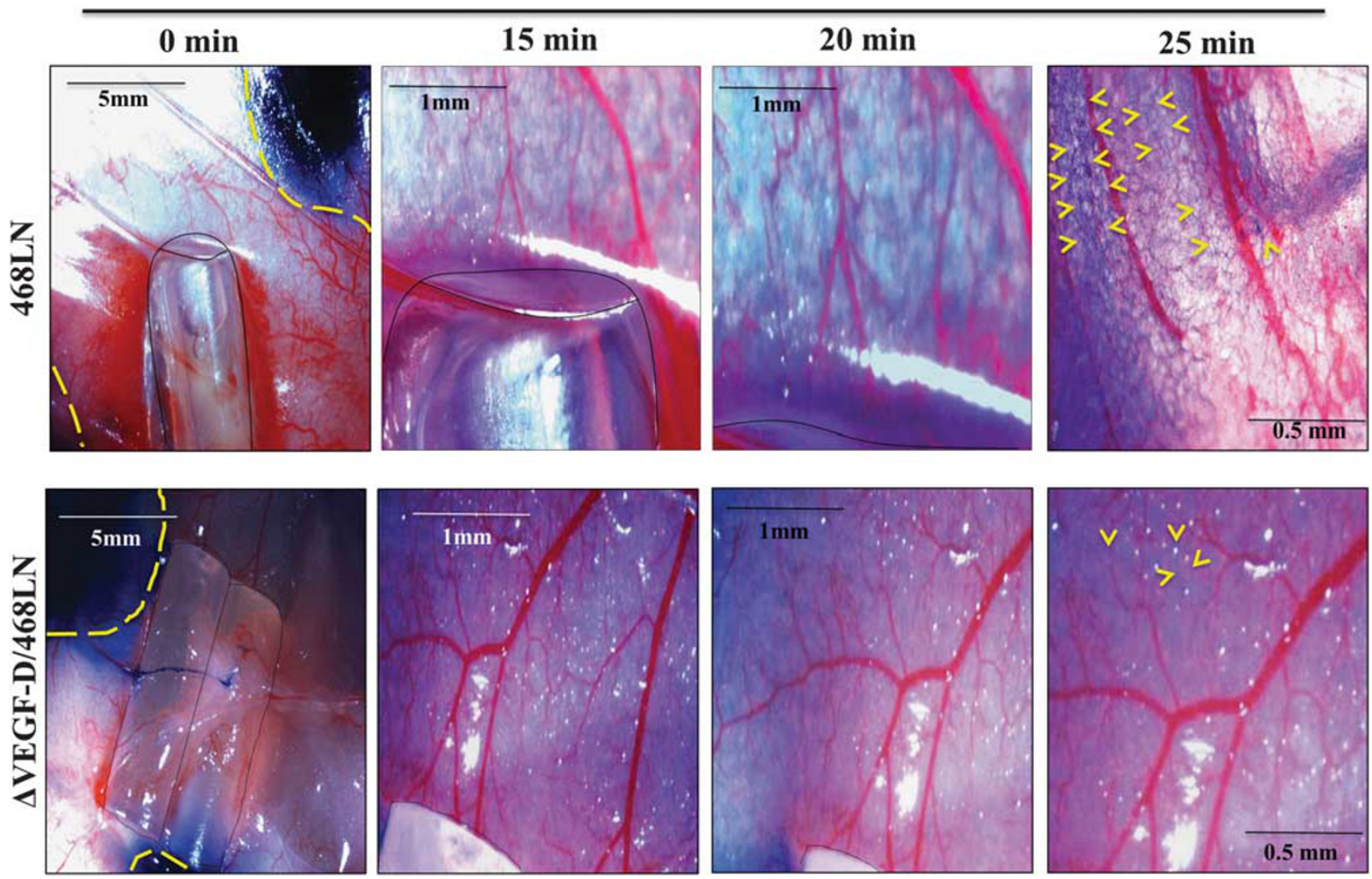

Figure 2 Evans Blue dye tracing. Dye injection site is outlined by yellow. Arrowhead showing density of blue lymphatic network was high and directed towards the open ends of angioreactors (black lining) containing 468LN cells (upper panel). In contrast, very poor dye infiltration indicative of lymphatic network formation was detected near the tubes (black lining) containing $\Delta$ VEGF-D/468LN cells (lower panel, arrowhead). Images captured with a dissection microscope are shown at different magnifications (see inset) at different time intervals. VEGF, vascular endothelial growth factor. 
VEGF-D and with FGF-2/VEGF served as respective positive controls for lymphangiogenesis and angiogenesis. Using these five types of implants, we quantified both lymphangiogenesis and angiogenesis with indirect and direct measurements in nude mice as outlined in Figure 1 and detailed in methodology.

b
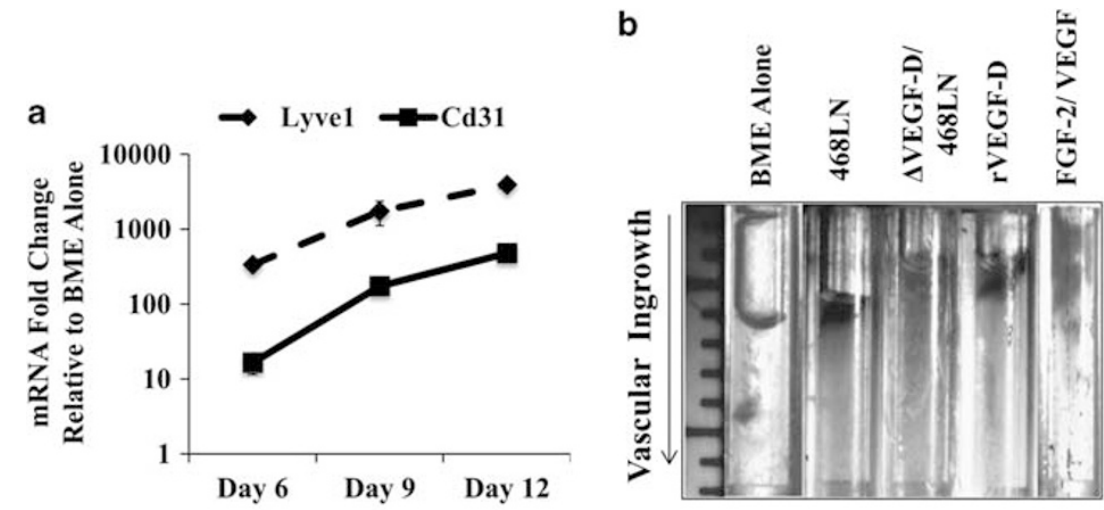

C
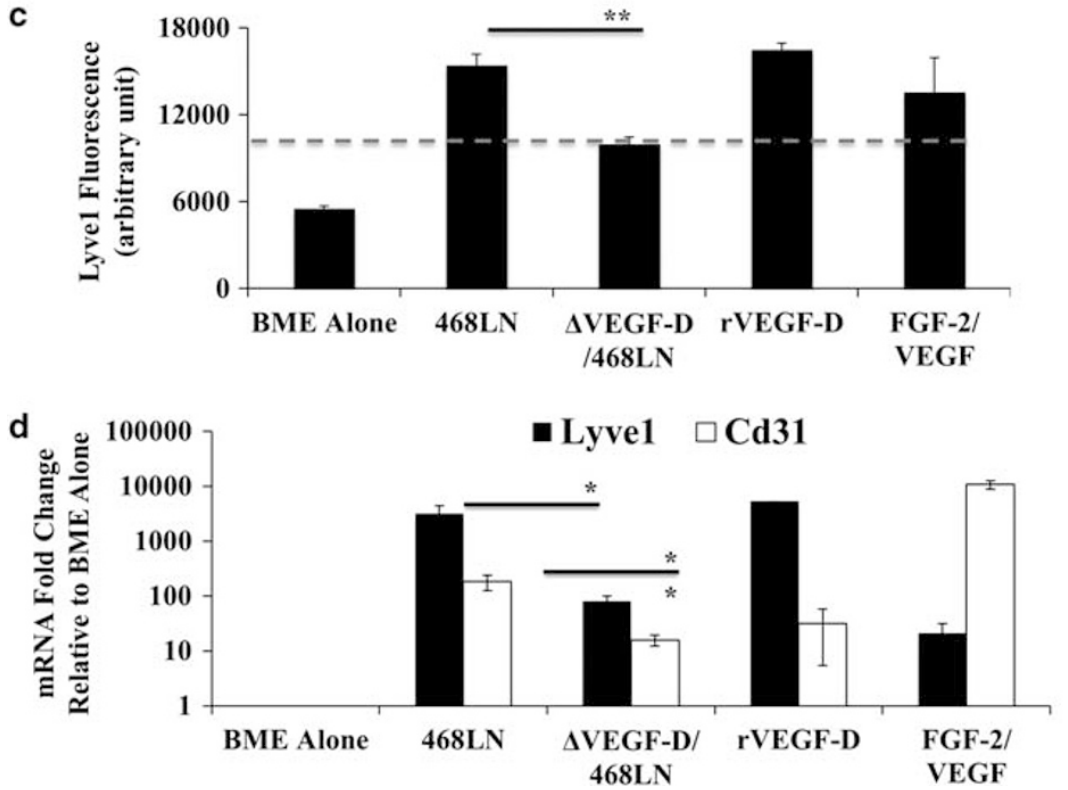

Figure 3 Lymphatic ingrowth assay and mRNA expression. (a) To determine the temporal kinetics of lymphatic network formation and stability, in a pilot study, four mice (for eachtime point, were killed on days 6, 9 and 12. Levels of lymphatic vessel endothelial hyaluronan receptor-1 (Lyve1) and Cd31 mRNA expression in cellular contents of angioreactor were measured and data presented relative to basement membrane extract (BME) alone. (b) Red blood cells were visibly higher in tubes containing 468LN cells than those with $\Delta$ VEGF-D/468LN cells. FGF-2/VEGF served as positive control for angiogenesis, rVEGF-D as positive control for lymphangiogenesis and BME as background control for both. (c) Cells isolated from tubes and labeled with mouse Lyve1 antibody to measure the relative incidence of lymphatic endothelial cells. Lyve1 expression is presented as raw fluorescence scores

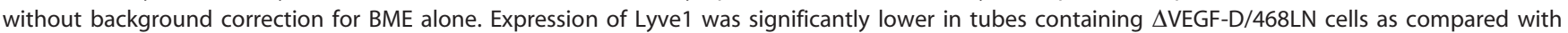
468LN, rVEGF-D and FGF-2/VEGF tubes. (d) Lyve1 and Cd31 mRNA expression was measured with cells collected from angioreactor and data presented relative to BME alone. Lyve1 expression was the highest with rVEGF-D, and Cd31 expression the highest with FGF-2/VEGF. VEGF-D knockdown markedly reduced the expression of both markers in $\Delta$ VEGF-D/468LN cells compared with $468 \mathrm{LN}$ cells. Bars in (b) and (c) represent mean ( $n=16) \pm$ s.e. ${ }^{*} P<0.05 ;{ }^{* *} P<0.005$. FGF, fibroblast growth factor; VEGF, vascular endothelial growth factor.

Figure 4 Direct measurement of lymphangiogenesis and angiogenesis with rVEGF-D in nude mice. (a) Representative sections of angioreactors showing cellular morphology in hematoxylin and eosin staining. Vascular-like networks can be seen in tubes with rVEGF-D (arrowheads). Images taken with $\times 10$ objectives scale bar represents $200 \mu \mathrm{m}$. (b) Immunofluorescent labeling for murine lymphatic vessel endothelial hyaluronan receptor-1 (Lyve1) or PROX-1 (Prox1) or Pdpn and Cd31 markers in serial frozen sections of angioreactors, containing rVEGF-D, served as positive control for lymphangiogenesis. In merged pictures, lymphatics are shown with arrowheads in white and blood vessels in yellow. A few lumens (white arrows) were observed. While significant angiogenesis as well as lymphangiogenesis was evident from all the markers, no significant overlap was noted between Lyve1 and Cd31 staining of endothelial cells. BME, basement membrane extract; DAPI, 4',6-diamidino-2-phenylindole; VEGF, vascular endothelial growth factor. 
a

\section{BME}

rVEGF-D

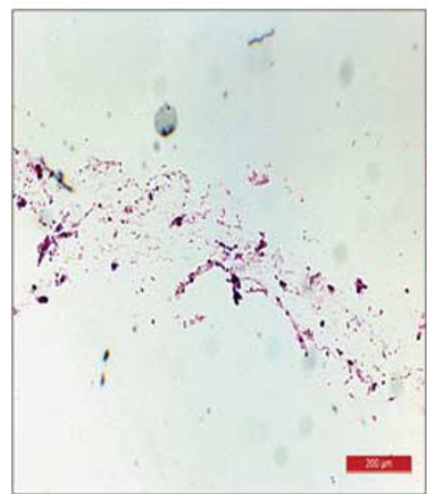

b
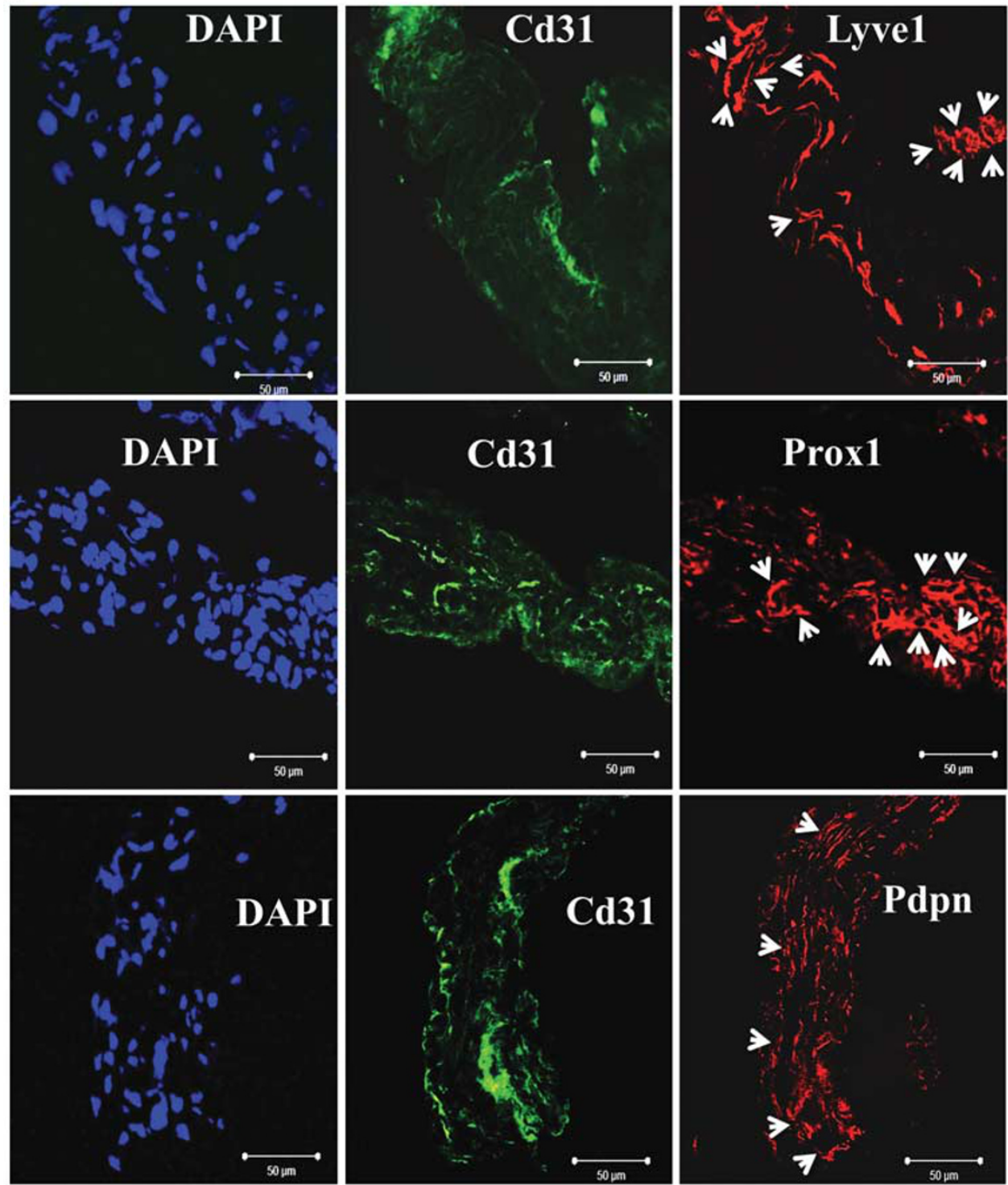

468LN

AVEGF-D/ 468LN 


\section{Indirect Measurements}

VEGF-D knockdown in tumor cells reduces the density of visible lymphatic network on the surface and ingrowth of lymphatics into the angioreactors

Blood vessels including blood capillaries are visibly red because of red cell content. Lymphatic networks are traceable with Evans Blue dye. An abundance of dye-stained lymphatic capillary network was visible around 468LN angioreactor implants (arrowhead) at 10-25 min after injection (Figure 2, upper panel). In contrast, in mice implanted with angioreactors containing $\Delta$ VEGF-D/468LN cells, Evans Bluemarked large lymphatics were visible, but lymphatic capillary network around angioreactors were not visible even at $25 \mathrm{~min}$ (Figure 2, lower panel, arrowhead).

To determine initiation of lymphangiogenesis and its temporal kinetics, we conducted a pilot study in a small number of mice. BME and 468LN served as respective negative and positive controls. One mouse per group was killed at each time points on days 6,9 and 12. Levels of angiogenesis and lymphangiogenesis were measured by quantifying Cd31 and Lyvel mRNA (Figure 3a). Significant lymphangiogenesis was detected as early as day 6 , increasing to a plateau between days 9 and 12 . Thus in the definitive experiments, we killed the mice on day 10 .

Red blood cell-containing vasculature within the angioreactors was evident once tubes were exposed. Tubes containing 468LN cells or rVEGF-D alone showed significantly detectable red blood cell content, the highest content observed in the tubes containing FGF-2/VEGF, serving as positive control for angiogenesis. VEGF-D knockdown in $468 \mathrm{LN}$ cells resulted in a marked reduction in red blood cell content (Figure 3b). Results from immunofluorescence assay for lymphangiogenesis marker Lyve1 (uncorrected for the background provided by BME alone, used as negative control) revealed that Lyve1-labeled murine LECs were recruited into the angioreactors during lymphangiogenesis. Lyvel signal was significantly greater in cells collected from $468 \mathrm{LN}$ angioreactor than $\triangle$ VEGF-D/468LN angioreactor (Figure 3c). As expected, the signal was the highest in BME containing rVEGF-D used as positive control for lymphangiogenesis.

\section{VEGF-D knockdown reduces the expression of both murine Lyve1 and Cd31 mRNA}

To quantify at mRNA levels indicative of the relative abundance of mouse lymphatics and blood vessels within the angioreactors, we took advantage of the mouse lymphatic endothelial marker Lyve1 and angiogenesis marker Cd31. As expected, implants with FGF-2/VEGF exhibited high Cd31 but very low Lyve1 mRNA, and those with rVEGF-D showed very high Lyve1 but low Cd31 mRNA, whereas implants with BME alone expressed very little of either mRNA. Finally, there was a significant reduction in both Lyve1 and Cd31 mRNA expression in cells collected from $\triangle$ VEGF-D/ 468LN-containing angioreactors, as compared with 468LN angioreactors (Figure $3 \mathrm{~d}$ ).

\section{Direct Measurements}

VEGF-D knockdown in $468 \mathrm{LN}$ cells reduces both lymphangiogenesis and angiogenesis within the angioreactors Hematoxylin and eosin-stained sections of angioreactor contents (Figure 4a) revealed very few cells in BME alone, whereas an abundance of cells inclusive of vascular-like networks was noted in angioreactors containing r-VEGF-D, 468LN or $\Delta$ VEGF-D/468LN cells. Dense vascular-like networks were noted in VEGF-D-containing angioreactors. All three antibodies (Lyve1 or Prox1 or Pdpn) showed specific staining of lymphatic network in sections of angioreactors containing r-VEGF-D, used as positive control for lymphangiogenesis. Blood vessels were stained with $\mathrm{Cd} 31$ in the same sections (Figure $4 \mathrm{~b}$ ). We directly measured the levels of lymphangiogenesis and angiogenesis in serial cryosections of angioreactors with dual immunolabeling for Lyve1 or Prox1 (sky blue) and Cd31 (red) or Pdpn (red) and Cd31 (sky blue), respectively, using the 'hotspots', as reported. The unit scores are the means of three hot spots (as detailed in the Materials and Methods) under each treatment condition, representing the incidence of marker-positive vascular-like structures (with or without complete lumens) per unit area.

High levels of tumor-associated lymphangiogenesis as well as angiogenesis occurred in 468LN-containing implants. Unsurprisingly, many lymphatic and blood vessels coexisted in intimate contact. Incidences of both vascular structures were significantly lower in $\triangle$ VEGF-D/468LN cell-containing implants (images shown in Figure 5a; quantitation in Figure 5b). No measurable angiogenesis or lymphangiogenesis was observed in BME-alone implants (data not shown). Some EGFP-tagged 468LN and $\triangle$ VEGF-D/468LN cells (green) were also noted in unlabeled angioreactor sections (Figure 5a). To test whether macrophages stained for Lyve1 as reported in some studies, ${ }^{28}$ some serial sections were also double-stained for F4/80 (red) and Lyvel (sky blue) (Figure 6). The results showed little or no Lyvel staining by F4/80-positive cells.

Therapy with COX-2 inhibitor reduces both lymphangiogenesis and angiogenesis within the angioreactor-containing C3L5 cells In 9-day-old implants of C3L5-containing angioreactors in $\mathrm{C} 3 \mathrm{H} / \mathrm{HeJ}$ mice, data revealed a very high incidence for both Lyve1- and Cd31-stained vascular structures in vehicle-treated mice, which was significantly reduced in Celecoxib-treated mice (Supplementary Figure 1). These results are highly consistent with our earlier report of inhibition of tumor growth, tumor-associated lymphangiogenesis and angiogenesis and spontaneous metastasis to the lymph nodes and the lungs in this tumor model following Celecoxib therapy. ${ }^{25}$

\section{DISCUSSION}

Here we describe a practical, sensitive and quantitative assay for measuring lymphangiogenesis in vivo within a short time frame of 9-10 days utilizing multiple approaches, including direct immunohistochemical identification and scoring of 


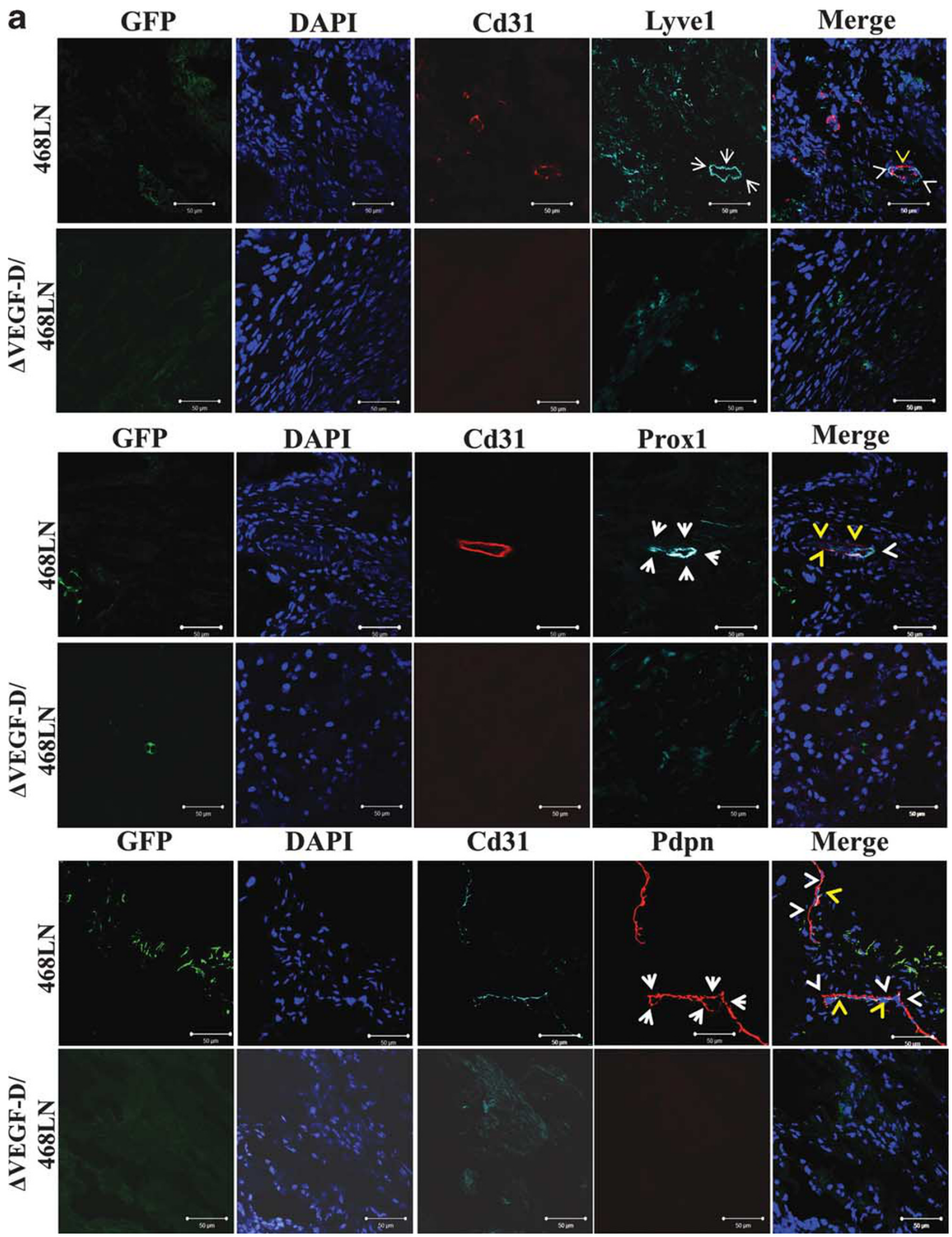

Figure 5 See next page for caption. 
b
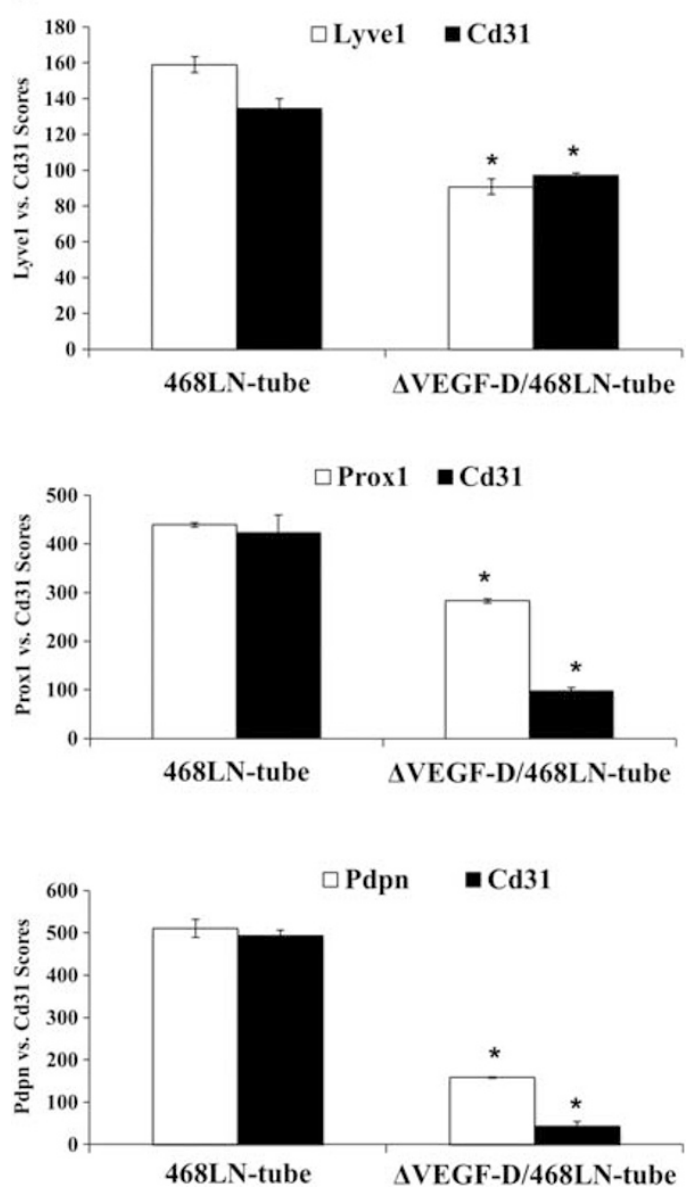

Figure 5 Measurements of lymphangiogenesis and angiogenesis in tubes containing $468 \mathrm{LN}$ and $\triangle \mathrm{VEGF}-\mathrm{D} / 468 \mathrm{LN}$ cells. (a) Immunofluorescent labeling for murine lymphatic vessel endothelial hyaluronan receptor-1 (Lyve1) or PROX-1 (Prox1) (sky blue) and Cd31 (red) or Pdpn (red) and Cd31 (sky blue) markers in serial frozen sections of angioreactors revealed significantly higher labeling for all markers in 468LN-containing sections (white arrowheads showing staining of either Lyve1 or Prox1 or $\mathrm{Pdpn}$ and yellow arrowheads showing $\mathrm{Cd} 31$ staining in merged pictures) compared with $\triangle \mathrm{VEGF}-\mathrm{D} / 468 \mathrm{LN}$ sections. Nuclei were stained with DAPI, 4',6-diamidino-2-phenylindole (DAPI) (royal blue). Some enhanced green fluorescent protein (EGFP)-tagged 468LN and $\triangle$ VEGF-D/468LN cells (green) were also visible. A few of the vessels show evident lumens (white arrows). Magnifications are shown as insets in the merged picture, scale bars representing $50 \mu \mathrm{m}$. (b) Quantification of 'hotspot' scores for Cd31 and Lyve1 or Prox1 or Pdpn made under $\times 40$ objective $(n=8$, using the mean of three hot spots from each of the eight angioreactors per group) \pm s.e., ${ }^{*} P=0.0001$. VEGF, vascular endothelial growth factor. The color reproduction of this figure is available on the Laboratory Investigation journal online.

lymphatic capillaries. In this assay, most of our experiments were conducted in nude mice using the MDA-MB-468LN cell line, a lymph node-metastasizing variant of the MDA-MB468 human breast adenocarcinoma cell line. The assay was further validated with a single experiment using a highly metastatic murine C3L5 breast cancer cell line in immunocompetent mice. In an earlier study, utilizing numerous other in vitro assays and tumor xenotransplants in nude mice, we have defined the multiplicity of mechanisms underlying the capacity of the 468LN cells in promoting lymphangiogenesis, lymphovascular invasion and lymphatic metastasis. ${ }^{8}$ We showed that they resulted from the dual overexpression of $\alpha 9 \beta 1$-integrin and its ligand VEGF-D by 468LN cells. The limitation of the above-mentioned tumor model is that it is time consuming for the xenograft to produce tumors of appreciable sizes to measure intratumoral lymphangiogenesis. In a syngeneic mouse model of breast cancer expressing both VEGF-C and VEGF-D, in which tumors grew rapidly and quickly metastasized to lymph nodes, the same results were achievable as early as 12 days. ${ }^{25}$ We show here that the current assay is equally applicable to this tumor model in immunocompetent mice tested at 9 days. However, xenografted human tumor models being best suited to preclinical testing of drugs, the present assay is ideally suited for such preclinical validation.

DIVAA is an excellent well-studied model to measure angiogenic factor-induced angiogenesis or its inhibition with angiostatic agents after implanting angioreactors in the mouse dorsal flank. ${ }^{23}$ This is an improvement on the matrigel plug assay as reported by us for tumor-associated angiogenesis, ${ }^{29}$ by containing the BME (or matrigel) in a silicon cylinder to direct the ingrowth of newly formed capillaries into the open end of the cylinder. DIVAA has been effectively applied to studies of both proangiogenic ${ }^{30}$ and antiangiogenic ${ }^{24}$ functions of various agents. We modified and applied this method to quantify lymphangiogenesis and coined the term DIVLA or directed in vivo lymphangiogenesis assay. This method is quick, highly quantitative and can be applied to a variety of studies, including preclinical testing of agents for their ability to block or promote lymphangiogenesis in vivo. As many compounds or tumor cells may have a dual effect on angiogenesis and lymphangiogenesis, as illustrated in this study in the case of VEGF-D, or 468LN or C3L5 cells, its capability for a simultaneous measurement of angiogenesis is an additional advantage. VEGF-D, although well known for its capacity to promote lymphangiogenesis by virtue of binding to VEGF-R3 expressed by lymphatic endothelial precursors, can also bind to VEGF-R2 expressed by vascular endothelial precursors to promote angiogenesis, explaining the dual effect in this study using either 468LN cells (expressing high levels of VEGF-D, but not VEGF-C or VEGF-A) or recombinant VEGF-D in the BME of implanted angioreactors. Knocking down VEGF-D in 468LN cells significantly abrogated both events. This was also the case with Celecoxib therapy in the case of C3L5 cells producing both VEGF-C and VEGF-D, resulting from COX-2 expression. As clearly demonstrated in our results, implants containing growth factor-reduced BME alone showed no sign of angiogenesis or lymphangiogenesis in any of the indirect or direct measurements in our assay, eliminating any role of the matrix in the implanted angioreactors. 

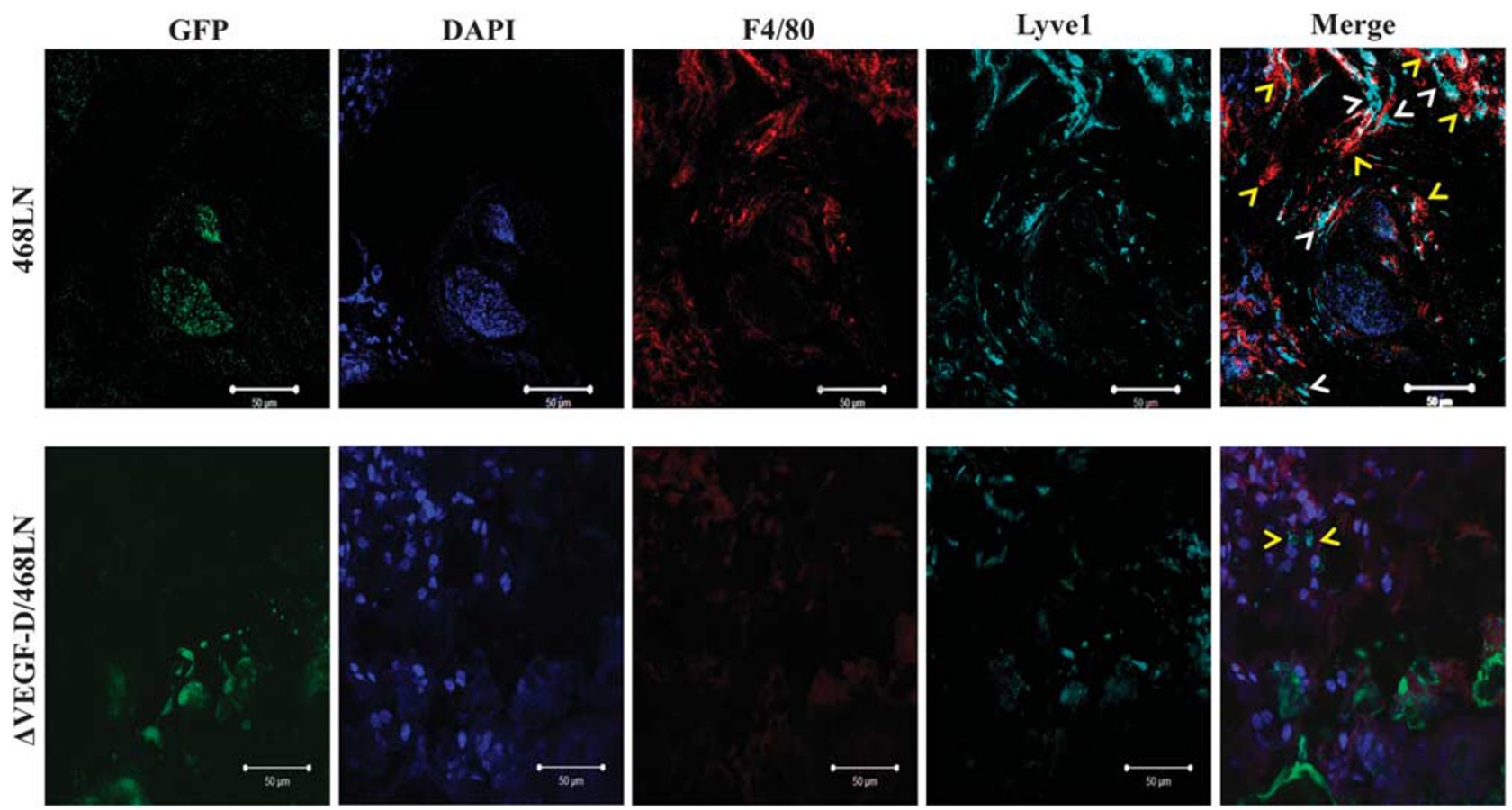

Figure 6 Dual immunostaining for lymphatic vessel endothelial hyaluronan receptor-1 (Lyve1) and F4/80. Immunofluorescent labeling for Lyve1 (sky blue) and F4/80 (red, macrophage marker) in frozen sections of angioreactors. Merged pictures (Lyve1, white arrowhead; F4/80, yellow arrowhead) revealed very little or no overlap between the two markers. Nuclei were stained with 4',6-diamidino-2-phenylindole (DAPI) (royal blue). Some enhanced

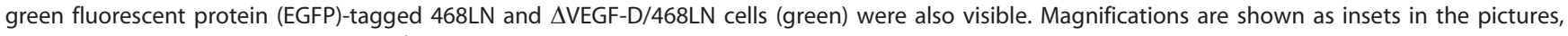
scale bars representing $50 \mu \mathrm{m}$. DAPI, 4',6-diamidino-2-phenylindole.

A simpler matrigel plug assay without the inclusion of angioreactors, in our hands, does not provide as robust results because of the undirected nature of the lymphatic vessel ingrowth, as well as a poorer containment of tumor cells within the implant. Inclusion of tumor cells in the BME within the angioreactor makes its application highly suited for studies of tumor biology and translational cancer research, as has been exploited with the DIVAA model. ${ }^{22,23}$ The role of tumor-associated lymphangiogenesis in lymphatic metastasis has been suggested by an association between tumoral expression of the lymphangiogenic factors VEGF-C or -D and lymphatic metastasis in many epithelial cancers including cancers of the prostate, ${ }^{31}$ stomach $^{32}$ and the colon. ${ }^{33}$ This has been validated by a direct association between intra- or peritumoral lymphangiogenesis with lymphatic invasion and metastasis in cancers of the breast, ${ }^{4,5}$ pharynx and larynx. ${ }^{3}$ Thus, this assay should have a broad application for preclinical testing of agents with potentials for prevention or intervention of lymphatic metastasis.

Multiple quantitative measurements and markers described in this assay make our model more robust and sensitive compared with the single measurement used for angiogenesis in the original DIVAA assay. ${ }^{23}$ We used a variety of approaches such as quantification of the Lyvel protein by immunofluorescence, Lyvel mRNA by qPCR from the extracted tissues and direct immunolabeling of frozen sections for multiple markers (Lyve1, Prox1 and Pdpn), all of which gave the same answer, eliminating the limitations of a single approach or a single marker. We found no evidence of Lyve1-stained endothelial linings also staining for $\mathrm{Cd} 31$ in our earlier study ${ }^{25}$ or this study. To exclude the possibility of Lyve1 staining by macrophages reported in some studies ${ }^{28}$ leading to overestimate LVD, we double-stained tissue sections with F4/80 and Lyve1 showing little or no overlap. Furthermore, use of $\operatorname{Prox}^{13}$ and $\operatorname{Pdpn}^{12}$ as additional markers provided very similar results.

This assay can be adapted to a direct testing of the drugs included in the BME along with tumor cells (with drugs with a long half-life), or of drugs given systemically to mice by the enteral or parenteral routes and still get the results in 10 days. There are many drugs that may have no direct effect on the process of lymphangiogenesis, but can block tumor-induced lymphangiogenesis by inhibiting pathways responsible for an upregulation of lymphangiogenic factors such as VEGF-C or VEGF-D produced by tumor cells. For example, expression of COX-2 was shown to upregulate VEGF-C or -D in breast cancer ${ }^{25,34}$ and lung cancer. ${ }^{35}$ In the case of breast cancer, this was primarily due to an activation of prostaglandin receptor EP4, so that both COX-2 inhibitor Celecoxib and the EP4 antagonist ONO-AE3-208 given orally were equally effective in blocking tumor-associated lymphangiogenesis and lymphatic metastasis in a COX-2-expressing mouse breast cancer model. ${ }^{25}$ In this study, using DIVLA we have 
reproduced the effects of Celecoxib therapy in the same tumor noted earlier with the matrigel plug assay. ${ }^{25}$

In conclusion, the DIVLA is an innovated version of the DIVAA method for quick and efficient measurements of lymphangiogenesis in combination with angiogenesis. It is highly practicable in laboratories housing nude mice, with training for implanting angioreactors. This implantation is relatively easier in young (6-8 weeks old) nude mice than in immunocompetent mice of similar age, which have a thicker and hairy skin requiring shaving or depilation. The method allows identification of new candidates that regulate lymphatic vasculature, a screening for pro- and antilymphangiogenic agents, and exploring specific mechanisms that underlie or differentially regulate the processes of lymphangiogenesis and angiogenesis. We believe that it presents advancement in vascular biology with the potential of identifying common as well a distinct progenitors and pathways for angiogenesis and lymphangiogenesis. In particular, it offers prospects for the design of strategies and drugs to manipulate the lymphatic system, and thereby intervene the processes of cancer metastasis and lymphedema.

Supplementary Information accompanies the paper on the Laboratory Investigation website (http://www.laboratoryinvestigation.org)

\section{ACKNOWLEDGEMENTS}

We thank Dr Lynne-Marie Postovit for technical advice, Dr Daniela Quail, Guihua Zhang, Tom Chrones and Mauricio Rodriguez for technical help, and the Ontario Institute of Cancer Research and the Canadian Breast Cancer Foundation (CBCF), Ontario Chapter, for grant support to PKL. MM is a fellow of The Translational Breast Cancer Research Unit (TBCRU).

\section{DISCLOSURE/CONFLICT OF INTEREST}

The authors declare no conflict of interest.

1. Cao Y. Opinion: emerging mechanisms of tumour lymphangiogenesis and lymphatic metastasis. Nat Rev Cancer 2005;5:735-743.

2. Alitalo K, Tammela T, Petrova TV. Lymphangiogenesis in development and human disease. Nature 2005;438:946-953.

3. Beasley NJ, Prevo R, Banerji S, et al. Intratumoral lymphangiogenesis and lymph node metastasis in head and neck cancer. Cancer Res 2002;62:1315-1320.

4. Van der Auwera I, Van den Eynden GG, Colpaert CG, et al. Tumor lymphangiogenesis in inflammatory breast carcinoma. A histomorphometric study. Clin Cancer Res 2005;11:7637-7642.

5. Bhattacharjee RN, Timoshenko AV, Cai J, et al. Relationship between cyclooxygenase-2 and human epidermal growth factor receptor 2 in vascular endothelial growth factor $\mathrm{C}$ up-regulation and lymphangiogenesis in human breast cancer. Cancer Sci 2010;101:2026-2032.

6. Makinen T, Jussila L, Veikkola T, et al. Inhibition of lymphangiogenesis with resulting lymphedema in transgenic mice expressing soluble VEGF receptor-3. Nat Med 2001;7:199-205.

7. Françoise Bruyère Melen-Lamalle $\mathrm{L}$, Blacher $\mathrm{S}$, Roland $\mathrm{G}$, et al. Modeling lymphangiogenesis in a three-dimensional culture system. Nat Methods 2008;5:431-437.

8. Majumder M, Tutunea-Fatan E, Xin X, et al. Co-expression of $\alpha 9 \beta 1$ integrin and VEGF-D confers lymphatic metastatic ability to a human breast cancer cell line MDA-MB-468LN. PLoS One 2012;7:e35094.

9. Nagy JA, Vasile E, Feng $D$, et al. Vascular permeability factor/vascular endothelial growth factor induces lymphangiogenesis as well as angiogenesis. J Exp Med 2002;196:1497-1506.
10. Jackson DG, Prevo R, Clasper S, et al. LYVE-1, the lymphatic system and tumor lymphangiogenesis. Trends Immunol 2001;22:317-321.

11. Cao $Y$, Linden $P$, Farnebo J, et al. Vascular endothelial growth factor $C$ induces angiogenesis in vivo. Proc Natl Acad Sci USA 1998;95: 14389-14394.

12. Ordóñez NG. D2-40 and podoplanin are highly specific and sensitive immunohistochemical markers of epithelioid malignant mesothelioma. Hum Pathol 2005;36:372-380.

13. Wigle JT, Harvey N, Detmar M, et al. An essential role for Prox 1 in the induction of the lymphatic endothelial cell phenotype. EMBO J 2002;21:1505-1513.

14. Stacker SA, Caesar C, Baldwin ME, et al. VEGF-D promotes the metastatic spread of tumor cells via the lymphatics. Nat Med 2001; 7:186-191.

15. Skobe $M$, Hawighorst $T$, Jackson DG, et al. Induction of tumor lymphangiogenesis by VEGF-C promotes breast cancer metastasis. Nat Med 2001;7:192-198.

16. Mandriota SJ, Jussila L, Jeltsch $M$, et al. Vascular endothelial growth factor-C-mediated lymphangiogenesis promotes tumour metastasis. EMBO J 2001;20:672-682.

17. Kopfstein L, Veikkola T, Djonov VG, et al. Distinct roles of vascular endothelial growth factor- $D$ in lymphangiogenesis and metastasis. Am J Pathol 2007;170:1348-1361.

18. Mancardi S, Stanta G, Dusetti N, et al. Lymphatic endothelial tumors induced by intraperitoneal injection of incomplete Freund's adjuvant. Exp Cell Res 1999;246:368-375.

19. Cao R, Björndahl MA, Religa P, et al. PDGF-BB induces intratumora lymphangiogenesis and promotes lymphatic metastasis. Cancer Cell 2004;6:333-345.

20. Cao R, Lim S, Ji H, et al. Mouse corneal lymphangiogenesis model. Nat Protoc 2011;6:817-826.

21. Oh SJ, Jeltsch MM, Birkenhäger $\mathrm{R}$, et al. VEGF and VEGF-C specific induction of angiogenesis and lymphangiogenesis in the differentiated avian chorioallantoic membrane. Dev Biol 1997; 188:96-109.

22. Martínez A, Vos M, Guédez L, et al. The effects of adrenomedullin overexpression in breast tumor cells. J Natl Cancer Inst 2002;94 1226-1237.

23. Guedez L, Rivera AM, Salloum R, et al. Quantitative assessment of angiogenic response by the directed in vivo angiogenesis assay. Am J Pathol 2003;162:1431-1439.

24. Seo DW, Li H, Guedez L, et al. TIMP-2 mediated inhibition of angiogenesis. An MMP-independent mechanism. Cell 2003;114: $171-180$.

25. Xin X, Majumder M, Girish GV, et al. Targeting COX-2 and EP4 to control tumor growth, angiogenesis, lymphangiogenesis and metastasis to the lungs and lymph nodes in a breast cancer model. Lab Invest 2012;92:1115-1128.

26. Vantyghem SA, Allan AL, Postenka $\mathrm{CO}$, et al. A new model for lymphatic metastasis. Development of a variant of the MDA-MB-468 human breast cancer cell line that aggressively metastasizes to lymph nodes. Clin Exp Metast 2005;22:351-361.

27. Rozic JG, Chakraborty C, Lala PK. Cyclooxygenase inhibitors retard murine mammary tumor progression by reducing tumor cell migration, invasiveness and angiogenesis. Int J Cancer 2001;93: 497-506.

28. Schledzewski K, Falkowski M, Moldenhauer $G$, et al. Lymphatic endothelium-specific hyaluronan receptor LYVE-1 is expressed by stabilin-1 +, F4/80 +, CD11b + macrophages in malignant tumours and wound healing tissue in vivo and in bone marrow cultures in vitro. Implications for the assessment of lymphangiogenesis. J Pathol 2006;209:67-77.

29. Jadeski LC, Lala PK. Nitric oxide synthase inhibition by $N(G)$-nitro-Darginine methyl ester inhibits tumor-induced angiogenesis in mammary tumors. Am J Pathol 1999;155:1381-1390.

30. Wang T, Ward Y, Tian L, et al. CD97, an adhesion receptor on inflammatory cells, stimulates angiogenesis through binding integrin counter receptors on endothelial cells. Blood 2005; 105:2836-2844.

31. Tsurusaki T, Kanda S, Sakai $\mathrm{H}$, et al. Vascular endothelial growth factor- $C$ expression in human prostatic carcinoma and its relationship to lymph node metastasis. $\mathrm{Br} J$ Cancer 1999;80: 309-313. 
32. Yonemura $\mathrm{Y}$, Endo $\mathrm{Y}$, Fujita $\mathrm{H}$, et al. Role of vascular endothelial growth factor $\mathrm{C}$ expression in the development of lymph node metastasis in gastric cancer. Clin Cancer Res 1999;7: 1823-1829.

33. Akagi K, Ikeda $\mathrm{Y}$, Miyazaki M, et al. Vascular endothelial growth factor$\mathrm{C}$ (VEGF-C) expression in human colorectal cancer tissues. Br J Cancer 2000;83:887-891.
34. Timoshenko AV, Chakraborty C, Wagner GF, et al. COX-2-mediated stimulation of the lymphangiogenic factor VEGF-C in human breast cancer. Br J Cancer 2006:94:1154-1163.

35. Su JL, Shih JY, Yen ML, et al. Cyclooxygenase-2 induces EP1- and HER-2/Neu dependent vascular endothelial growth factor-C upregulation. A novel mechanism of lymphangiogenesis in lung adenocarcinoma. Cancer Res 2004;64:554-564. 